\title{
DIVERSIFICACIÓN DE CARTERAS DE INVERSIÓN CON CRIPTOMONEDAS
}

\author{
DIVERSIFICATION OF INVESTMENT PORTFOLIOS WITH CRYPTOCURRENCIES
}

Pedro Pablo Chambi Condori Universidad Nacional Jorge Basadre Grohmann Tacna, Perú

ORCID: https://orcid.org/0000-0002-8628-6825 Correo electrónico: pchambic@unjbg.edu.pe

\section{RESUMEN}

Objetivo: Organizar en forma experimental carteras de inversión con los criptoactivos bajo la teoría moderna de estructuración de portafolios de inversión de Markowitz. Método: La investigación fue del tipo cuantitativa y experimental, abarcando el proceso de estructuración de portafolios de inversión con criptomonedas bajo la teoría de Markowitz, mediante el modelo de programación no lineal cuantitativa. Resultados: Se pudo observar que la cotización más pronunciada se presentó en el periodo comprendido entre 2017 a 2018. Una rentabilidad mayor le corresponde a Ethereum, seguido de Bitcoin y de Ripple; consecuentemente, Ethereum es el que tiene el mayor indicador de volatilidad. La opción de cartera eficiente se obtuvo cuando se invirtió el 70\% en Bitcoin, 14\% en Ethereum, $6 \%$ en Ripple y $10 \%$ en Thether y, como efecto de la diversificación de carteras de inversión, se verifica el comportamiento inverso de la volatilidad. Conclusiones: En el estudio en referencia, se demostró el efecto del número de activos en la conformación del portafolio de inversiones en la reducción de la volatilidad y las opciones que provee el mapa de la frontera eficiente para que los inversionistas opten por las opciones que calcen mejor con sus expectativas de riesgo y rentabilidad.

Palabras clave: Criptoactivos; innovación financiera; portafolios de inversión; optimización de portafolios.

\begin{abstract}
Objective: To experimentally organize investment portfolios with crypto assets under the modern theory of investment portfolio structuring of Markowitz. Method: The research was quantitative and experimental, explaining the process of structuring investment portfolios with cryptocurrencies under Markowitz's theory. Results: It was observed that the most pronounced trading period occurred from 2017 to 2018, where a higher profitability corresponds to Ethereum, followed by Bitcoin and Ripple, consequently Ethereum with the highest indicator volatility. The efficient portfolio option was obtained when $70 \%$ was invested in Bitcoin, $14 \%$ in Ethereum, $6 \%$ in Ripple and $10 \%$ in Thether and as an effect of the diversification of investment portfolios, the inverse behavior of volatility was verified. Conclusions: The study in reference demonstrated the effect of the number of assets in the formation of the investment portfolio in reducing volatility, and the options provided by the map of the efficient frontier for investors to opt for the options that better match the risk and return expectations.
\end{abstract}

Keywords: Cryptoassets; financial innovation; investment portfolios; portfolio optimization. 


\section{INTRODUCCIÓN}

En el contexto de la evolución de la tecnología blockchain y al lado de la cuarta revolución industrial en auge que tiene profundos impactos en la economía, entre ellos la incursión de nuevos instrumentos financieros y tecnológicos que inspiran a una nueva generación de inversionistas que buscan medios innovadores, las criptomonedas se han convertido en la mejor opción para la estructuración de sus carteras de inversión con la esperanza de obtener mejores retornos a determinados niveles de riesgo diversificable.

Los criptoactivos, llamados activos digitales, están atrayendo a inversionistas que buscan obtener rentabilidades altas, asumiendo riesgos también altos. Una de las reglas primordiales en la gestión de carteras de inversión con activos financieros, consiste en la elección de activos que aporten mayores beneficios a la cartera de inversiones y permitan a los inversionistas tener mejores opciones de diversificar su cartera con alternativas que les permitan lograr los niveles de rentabilidad esperados.

El objetivo central del trabajo es analizar, desde la perspectiva cuantitativa, la conformación de carteras de inversión con la inclusión de activos financieros denominados criptomonedas, bajo la teoría de selección de portafolio de inversiones de Markowitz (1959) y optimización de carteras de inversión bajo el modelo de Sharpe (2003). Asimismo, mostrar los efectos de la diversificación en la volatilidad de la rentabilidad esperada de una cartera de inversiones; como también, la correlación dinámica que presentan los activos en el transcurso del tiempo en estudio, bajo el supuesto de que las series de tiempo de los precios de los criptoactivos son del tipo autoregresivo y de estacionariedad condicionada. Finalmente, obtener un mapa de frontera eficiente de las diversas opciones de inversiones a fin de que los inversionistas tengan oportunidad de elegir de entre una diversidad de opciones, las que mejor calcen con sus expectativas de ganancia esperada y el riesgo asumido.

En relación con el tema de estructuración, diversificación y optimización de carteras de inversión con criptoactivos, se ha revisado la literatura publicada durante el periodo de 2017 a 2020 y la clásica relacionada a la organización de carteras de inversión (Ver Tabla 1). El artículo de Liu, Wang, Ahmad y Ma (2020) investiga el impacto de la diversificación con la adición de cinco criptomonedas a cuatro carteras de activos tradicionales en el periodo que

Tabla 1

Resumen de la revisión de las referencias sobre la construcción de portafolios de inversión

\begin{tabular}{|c|c|c|}
\hline Autores & Título del artículo/ Título de libro & Resultados \\
\hline Liu et al. (2020) & $\begin{array}{l}\text { Portfolio optimization in the era of digital fi- } \\
\text { nancialization using cryptocurrencies }\end{array}$ & $\begin{array}{l}\text { El estudio de optimización de carteras de inversión usando } \\
\text { criptomonedas en el periodo } 2015 \text { a } 2019 \text { con cuatro carte- } \\
\text { ras de activos clásicos }\end{array}$ \\
\hline Holovatiuk (2020) & $\begin{array}{l}\text { Cryptocurrences as an asset class in Portfo- } \\
\text { lio optimization }\end{array}$ & $\begin{array}{l}\text { El artículo analiza a las criptomonedas como instrumentos } \\
\text { de inversión, bajo la teoría moderna y posmoderna }\end{array}$ \\
\hline Can y Lagasse (2019) & $\begin{array}{l}\text { Cryptocurrencies: applications and invest- } \\
\text { ment opportunities }\end{array}$ & $\begin{array}{l}\text { El artículo examina a las criptomonedas como oportunida- } \\
\text { des de inversión }\end{array}$ \\
\hline Andrianto y Diputra (2018) & $\begin{array}{l}\text { The effect of Cryptocurrency on Investment } \\
\text { Portfolio Effectiveness }\end{array}$ & $\begin{array}{l}\text { El artículo aborda la aparición de la tecnología financiera } \\
\text { en los últimos } 10 \text { años y las criptomonedas ya formando } \\
\text { carteras de inversión }\end{array}$ \\
\hline Cortez at al (2018) & $\begin{array}{l}\text { Bitcoin: Its influence on the Global World and } \\
\text { its Relationship with the stock exchange }\end{array}$ & $\begin{array}{l}\text { El artículo examina el impacto de Bitcoin en el mercado de } \\
\text { acciones en la organización de carteras de inversión }\end{array}$ \\
\hline Jang y Lee (2017) & $\begin{array}{l}\text { An Empirical Study on Modeling and Predic- } \\
\text { tion of Bitcoin Prices With Bayesian Neural } \\
\text { Networks Based on Blockchain Information }\end{array}$ & $\begin{array}{l}\text { El artículo mediante un análisis experimental aborda la pro- } \\
\text { yección de precios del Bitcoin }\end{array}$ \\
\hline Sharma (2017) & $\begin{array}{l}\text { Build a Portfolio of Cryptocurrencies using } \\
\text { Modern Portfolio Theory }\end{array}$ & $\begin{array}{l}\text { El artículo desarrolla la metodología de la teoría moderna } \\
\text { de organización de carteras de inversión utilizando cripto- } \\
\text { monedas }\end{array}$ \\
\hline Damodaran (2012) & Investment Valuation & Aborda herramientas y técnicas de valoración \\
\hline Copeland y Weston (1988) & Financial Theory and Corporate Policy & $\begin{array}{l}\text { El texto aborda las herramientas y técnicas para determinar } \\
\text { el valor de cualquier activo }\end{array}$ \\
\hline Markowitz (1959) & $\begin{array}{l}\text { Portfolio Selection: Efficient Diversication of } \\
\text { Investments }\end{array}$ & $\begin{array}{l}\text { La teoría moderna cuantitativa de organización y optimi- } \\
\text { zación de carteras de inversión }\end{array}$ \\
\hline
\end{tabular}

Fuente: Elaboración propia con base en Scimago Journal \& Country Rank (2020) 
corre entre noviembre de 2015 hasta noviembre de 2019, encontrando resultados sobre cómo la diversificación aumentó la rentabilidad en la mayoría de los casos, redujo la volatilidad de la cartera en todas las opciones de inversión y también proporcionó mayores rendimientos en comparación con las carteras tradicionales para el mismo nivel de riesgo. Este estudio también reveló que los resultados podrían mejorar las ventas al descubierto. Finalmente, el estudio llevó a los investigadores a la conclusión de que la adición de múltiples criptomonedas en una cartera proporciona mejores resultados en la diversificación, y que Ethereum ofrece una mejor oportunidad de diversificación en comparación con Bitcoin.

Por otro lado, Holovatiuk (2020) realizó el estudio sobre criptomonedas para analizar si estos instrumentos pueden calificarse como una clase de activos y qué tipo de beneficios pueden aportar a las carteras del inversor. El estudio en referencia concluye que las criptomonedas satisfacen relativamente por completo los siete requisitos de activos, los cuales son: agregación estable, capacidad de inversión, homogeneidad interna, heterogeneidad externa, utilidad esperada, habilidad de selección y acceso rentable. También encontró que los activos criptográficos tienen propiedades de diversificación. Además, Andrianto y Diputra (2018), en un estudio de organización de portafolios de inversión con criptoactivos, analizaron la implicancia de adición de criptomonedas en carteras de inversión conformadas por monedas extranjeras, materias primas, acciones y ETF; utilizaron a Bitcoin, Riple y Litecoin, obteniendo resultados en la diversificación, la reducción en la volatilidad de la cartera y mejores rendimientos. Sharma (2017), en su análisis, propuso la organización de portafolios de inversión bajo el modelo basado en la medida de Omega y lo aplica con cuatro portafolios de inversión conformados por criptoactivos.

La teoría moderna de carteras de inversión de Markowitz (1959) es una referencia básica que describe con detalle el procedimiento a seguir en la organización de carteras de inversión con diversos tipos de activos, así se ha hecho notar a través de las anteriores referencias. Además, se ha adicionado a las criptomonedas como un activo componente de una cartera de inversión, por supuesto buscando maximizar la rentabilidad y minimizando la volatilidad de la cartera de inversiones. Por otro lado, el índice de Sharpe (2003) permite ubicar una opción de inversión dentro de un grupo de opciones de carteras de inversión que se obtienen en el proceso de organización de carteras con los criptoactivos, haciendo variar la cantidad de activos de los que está compuesta una cartera y pesos en una cartera de inversiones. En la Tabla 1, se presenta la revisión de la literatura relacionada con la organización de carteras de inversión con criptoactivos.
Las hipótesis que han guiado la investigación son las que se sustentan en la teoría moderna de estructuración de carteras de inversión y en la teoría de gestión de las mismas. Estas han sido formuladas en la forma que se enuncia a continuación:

- Hipótesis 1: El valor esperado de la rentabilidad de una cartera de inversiones organizada con activos digitales viene explicada por la proporción de activos que incluye una cartera y la volatilidad individual de cada uno de ellos.

- Hipótesis 2: La gestión del número de componentes en la organización de una cartera de inversiones con activos digitales tiene incidencia en el grado de volatilidad de una cartera de inversiones.

\section{MATERIAL Y MÉTODOS}

La investigación fue del tipo cuantitativa y experimental, explicando el proceso de estructuración de portafolios de inversión con criptomonedas bajo la teoría de Markowitz. Fue experimental, ya que se realizó la manipulación de la variable: número de componentes de cuatro portafolios organizados; esta manipulación de componentes del portafolio tiene impacto en la magnitud de la volatidad, ilustrada en la Figura 4.

Para la ejecución del estudio se seleccionó cinco principales criptomonedas que tienen mayor preferencia en el mercado, entre ellas Bitcoin, Ethereum, Ripple XRP, Litecoin y Thether, y con el insumo de estadística de precios diarios desde 7 de agosto de 2015 hasta 11 de noviembre de 2020. Junto a la ecuación siguiente se expresa la ganancia diaria de cada uno de los criptoactivos, $R_{i}=100 * \ln \left(\frac{P_{t}}{P_{t-1}}\right)$, en donde $P_{t}$ expresa el precio del activo financiero en el tiempo " $\mathrm{t}$ " y $P_{t-1}$ es el valor del criptoactivo en el tiempo " $\mathrm{t}-1$ ", para luego obtener la esperanza matemática de cada uno de los criptoactivos que da como derivado la rentabilidad de una cartera conformada por " $\mathrm{n}$ " activos: $E\left(R_{c}\right)=\sum_{i=1}^{n} X_{i} R_{i}$ y el riesgo de la cartera se calcula con la ecuación siguiente: $\sigma_{c}=\sqrt{\sum_{j=1}^{n} \sum_{i=1}^{n} W_{i} W_{j} \operatorname{COV}\left(R_{i}, R_{j}\right)}$; finalmente se obtiene un mapa de frontera eficiente para las diversas alternativas de inversiones, a fin de que los estructuradores de carteras de inversión tengan la oportunidad de seleccionar la mejor alternativa que satisfaga los resultados esperados por parte de los inversionistas, y mostrar la correlación dinámica que presentan en el tiempo los criptoactivos que intervienen en la construcción de los portafolios de inversión.

Para el tratamiento de la frontera eficiente, se invoca al modelo de optimización de la ganancia de un portafolio de inversiones, buscando minimizar su riesgo. A continuación, se presenta la ecuación matemática de 
Markowitz (1959) que describe el comportamiento de un portafolio organizado:

$$
\text { Maximización: } E\left(R_{t}\right)=\sum_{i=1}^{n} w_{i} E\left(R_{i}\right)
$$

Relacionado a las siguientes restricciones:

$$
\begin{gathered}
\sigma_{t}^{2}=\sum_{i=1}^{n} \sum_{j=1}^{m} x_{i} x_{j} \sigma_{i} \sigma_{j} \rho_{i j} \leq \sigma_{0}^{2} \\
\sum_{i=1}^{n} w_{i}=1, w_{i} \geq 0, \text { en donde }(i=1, \ldots, n)
\end{gathered}
$$

El modelo arriba presentado, describe el comportamiento de optimización de un portafolio de inversiones con " $n$ " activos, con ganancia esperada de $E\left(R_{t}\right)$. En donde $w_{i}$ denota el peso de cada uno de los componentes del portafolio de inversiones y $\sigma_{t}^{2}$ describe la volatilidad del portafolio organizado, utilizando para la experiencia el modelo de programación no lineal cuantitativa de maximización de la rentabilidad y minimización de riesgo.

Para modelar la minimización de la volatilidad y de la ganancia esperada por el portafolio de inversiones, a continuación se expresa el modelo matemático de Markowitz (1959) que describe el propósito de minimizar la volatilidad y maximizar la ganancia esperada del portafolio de inversiones.

$$
\begin{gathered}
\text { Min } \sigma_{t}^{2}=\sum_{i=1}^{n} \sum_{j=1}^{n} x_{i} x_{j} \sigma_{i} \sigma_{j} \rho_{i j} \\
\text { Sujeto a restricción de }: \\
E\left(R_{t}\right)=\sum_{i=1}^{n} w_{i} E\left(R_{i}\right) \geq R_{0} \\
\sum_{i=1}^{n} w_{i}=1, w_{i} \geq 0, \text { en donde }(i=1, \ldots, n)
\end{gathered}
$$

En el modelo presentado en líneas arriba, el $R_{0}$ es la ganancia mínima exigida.

La optimización de cartera de inversiones se encuentra utilizando la ecuación de Sharpe, tal cual se enuncia en la ecuación $S I=\frac{\left(E\left(R_{p}\right)-R_{f}\right)}{\sigma_{p}}$, en donde $\mathrm{E}(\mathrm{Rp})$ : rentabilidad de la cartera, Rf es la tasa no afectada por el riesgo y $\sigma_{p}$ describe el riesgo de la cartera.

\section{Proceso de estructuración de carteras de inversión con criptoactivos}

El proceso de construcción de carteras de inversión con diversas opciones responde a la necesidad de examinar varias alternativas que requieren los potenciales inversionistas, decantando su decisión en función de la ganancia esperada y el riesgo de cada alternativa.

La técnica utilizada para la selección de componentes de un portafolio de inversiones es el análisis técnico de series de tiempo de los diversos activos sobre los que se tienen interés los inversionistas.
Las fases que comprende la toma de decisiones de inversión presentan tres componentes principales: (1) la búsqueda de información que se enfrenta a superar diversas manifestaciones de sesgos de información, anclaje, autoridad, descuento hiperbólico, exceso de confianza, efecto halo, prueba social, (2) elección y contratación de la cartera, (3) seguimiento del producto que consiste en vigilar el comportamiento de la inversión seleccionada. El proceso de organización de portafolios de inversión con criptoactivos comprende los aspectos que se desarrollan a continuación:

\section{Política de inversión}

Consiste en focalizar los objetivos del inversionista y la cantidad de riqueza que estaría dispuesto a comprometer en la inversión. Como existe una relación positiva entre el riesgo y la ganancia esperada para la estrategia de inversiones debidamente analizadas, el proceso concluye con la identificación de criptoactivos que han de incluirse en la cartera de inversiones. En este contexto, según Lopes (1987) en cualquier situación el nivel de aspiración podría tener al menos orígenes diferentes: (1) La evaluación directa del valor esperado de lo que es seguro o razonable, (2) la influencia directa del contexto de las diversas alternativas que existen e (3) influencias externas.

\section{Análisis de criptoactivos}

En el proceso, el segundo aspecto consiste en llevar a cabo el análisis técnico y fundamental de cada uno de los valores sobre los que se tiene interés, lo que implica estudiar desde la perspectiva financiera el comportamiento histórico de varios valores candidatos. En este contexto, los analistas de valores emplean técnicas tales como la de análisis técnico, a quienes se les conoce con el nombre de analistas técnicos, y la de análisis fundamental, quienes son conocidos como fundamentalistas. El análisis técnico comprende el análisis histórico de los precios del mercado de criptoactivos, pronosticando su ocurrencia de variación de precios en el futuro, cuyos orígenes recuerdan a su creador Charles Dow.

\section{Construcción de una cartera}

La construcción de portafolios de inversión consiste en escoger a los activos que han de conformar un portafolio de inversiones, teniendo en cuenta la ganancia esperada que muestra al potencial inversionista y el riesgo que está dispuesto a aceptar para un determinado nivel de ganancia esperada, todo esto bajo el concepto del modelo cuantitativo CAPM, también llamado modelo de fijación de precios de activos de capital. 


\section{Revisión de la cartera}

Esta cuarta etapa del proceso de construcción de portafolios de inversión, en realidad se ocupa de la gestión del portafolio, la cual implica ir vigilando en el tiempo su comportamiento en términos de ganancia y riesgo esperados. En esta etapa el gestor de la cartera puede tomar la decisión de mantener la cartera, liquidar la inversión, incluir otros activos dentro del portafolio orientado por el objetivo de maximizar la ganancia y la minimización del riesgo. El inversionista en esta etapa enfrenta los sesgos de descuento hiperbólico, statu quo y coste hundido.

\section{Medición del desempeño del portafolio}

Bajo el enfoque de utilidad esperada, es la etapa en donde se controla la rentabilidad que viene obteniendo el portafolio y se observa sus perspectivas de crecimiento, la ganancia esperada y el impacto del riesgo del mercado en relación con la ganancia esperada.

\section{RESULTADOS}

En esta parte del estudio, en primer lugar, se exhiben los resultados descriptivos de los criptoactivos con los que se han organizado 12 opciones de portafolios de inversión $y$, posteriormente, el impacto de la diversificación en la volatilidad del portafolio. La Figura 1, siendo el primer elemento descriptivo, muestra la evolución histórica de los cinco criptoactivos, en cada una de ellas se exhiben las más altas cotizaciones en el periodo 2017 a 2018, y cotizaciones con tendencia bajista en el periodo de 2019 a 2020. La Figura 1 se obtuvo con la data histórica de cotizaciones diarias contadas desde el 7 de agosto 2015 hasta el 11 de noviembre de 2020.
BITCOIN

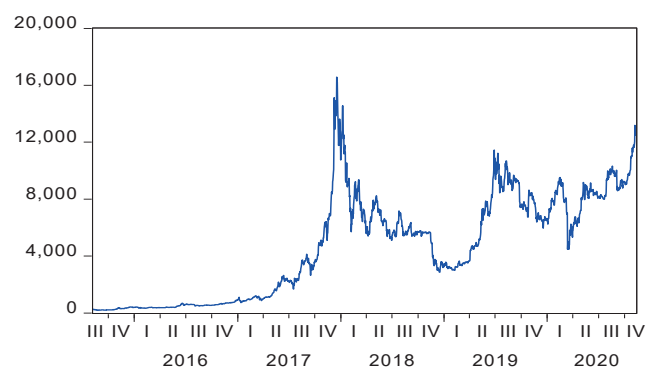

LITECOIN

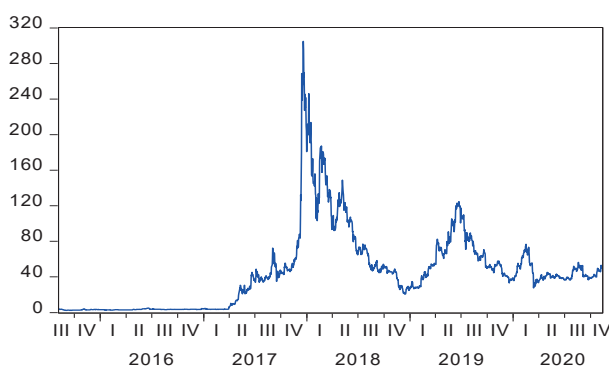

TETHER

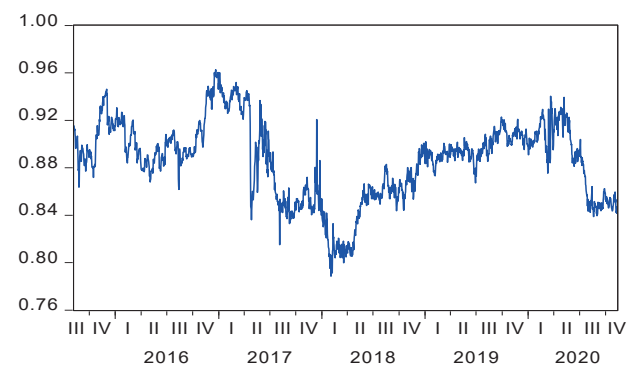

ETHEREUM

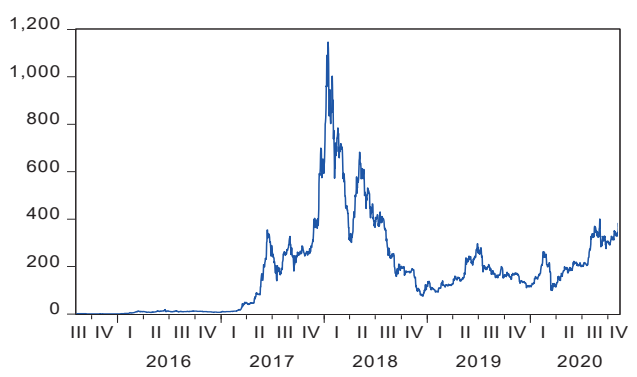

RIPPLE

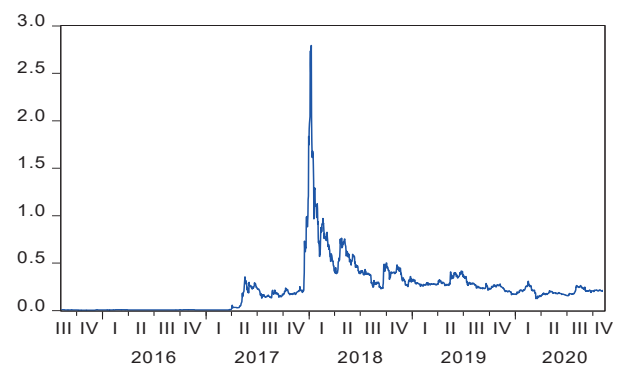

Figura 1. Serie histórica de cotización de criptomonedas 2015-2020

Fuente: Elaboración propia con base en en Investing.com (2021) 
El segundo elemento descriptivo es la matriz de correlación, la cual se presenta en la Tabla 2, dando información del grado de relación que mantuvieron entre sí los cinco criptoactivos; por ejemplo, el Bitcoin mostró una relación moderada con otros activos, mientras que el activo Theter sostuvo una relación indirecta con los otros cuatro activos.

De la información mostrada en la Tabla 2, se constata relaciones moderadas y directas entre los activos y rela- ción indirecta del activo Thether con los demás activos, situación que se visualiza también en la Figura 1, la cual describe con movimientos de cotización alcista del periodo 2017 a 2018 y desde entonces hasta 2020 comportamientos bajistas y alcistas en corto plazo.

La Figura 2 describe el comportamiento histórico de la ganancia diaria de los activos incluidos en el portafolio. Dicha Figura muestra periodos de alta volatilidad, como

Tabla 2

Matriz de correlación con criptomonedas

\begin{tabular}{lrrrrr}
\hline \multicolumn{1}{c}{ ACTIVOS } & BITCOIN & ETHEREUM & LITECOIN & RIPPLE & THETHER \\
\hline BITCOIN & 1.0000 & 0.7221 & 0.6149 & 0.6149 & -0.4254 \\
ETHEREUM & 0.7221 & 1.0000 & 0.8865 & 0.8516 & -0.7216 \\
LITECOIN & 0.7626 & 0.8865 & 1.0000 & 0.8503 & -0.5838 \\
RIPPLE & 0.6149 & 0.8516 & 0.8503 & 1.0000 & -0.5652 \\
THETHER & -0.4254 & -0.7216 & -0.5838 & -0.5652 & 1.0000 \\
\hline
\end{tabular}

Fuente: Elaboración propia con base en Investing.com (2021)
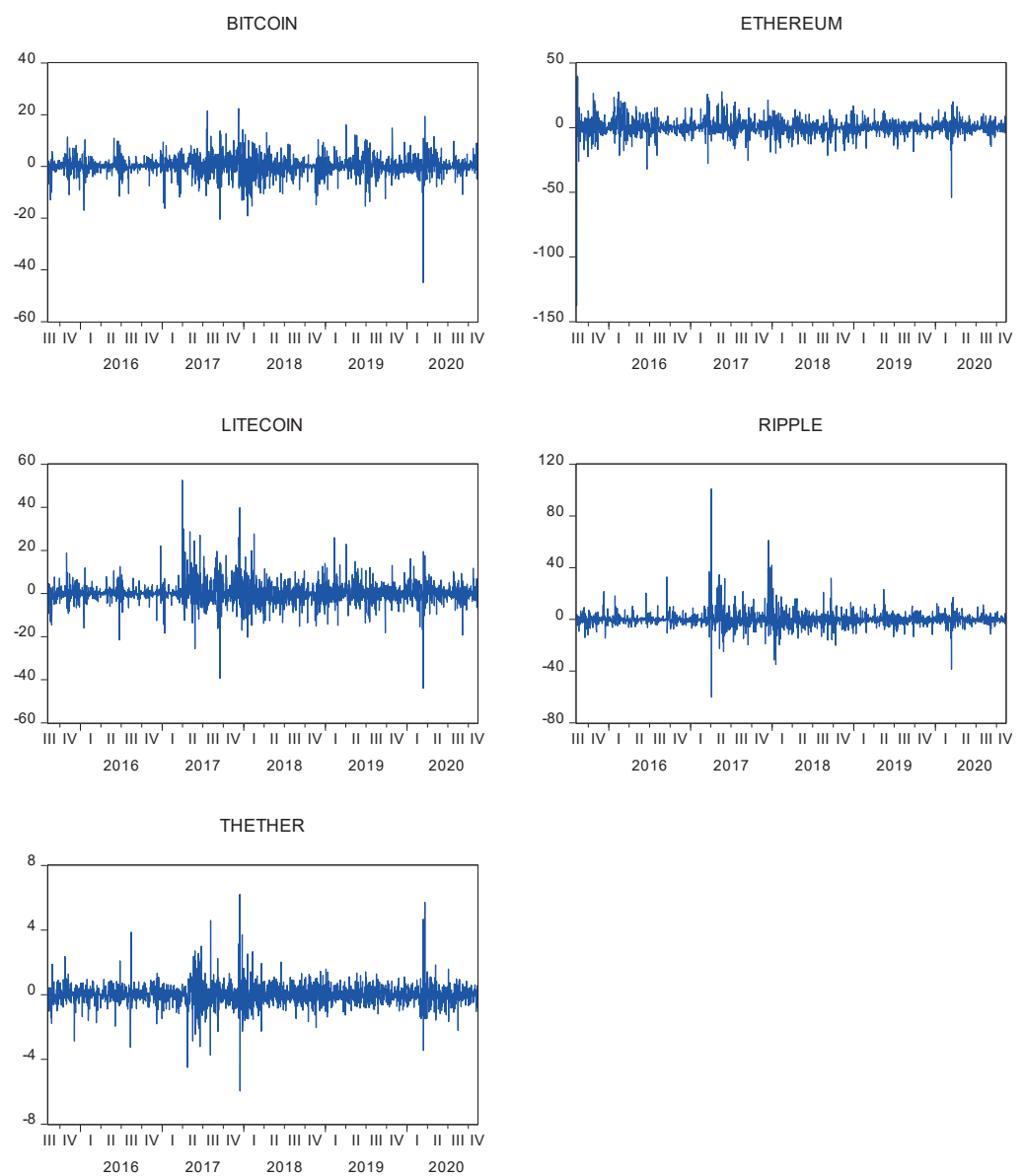

Figura 2. Volatilidad de la rentabilidad diaria en criptomonedas

Fuente: Elaboración propia con base en Investing.com (2021) 
el periodo 2017 a 2018, como es el caso que ocurrió con Bitcoin, en donde se constata la variación de la volatilidad de la rentabilidad diaria entre $+20 \%$ a $-20 \%$. La volatilidad de Ethereum, que es el activo que manifiesta mayor rentabilidad de entre los cinco activos en el periodo analizado, varía de $30 \%$ a -30\%; por otro lado, Ripple y Litecoin describen volatilidades que van desde $+20 \%$ a $-20 \%$.

La Figura 2 muestra la evolución histórica de la ganancia diaria de cada uno de los criptoactivos con los que se ha conformado el portafolio de inversiones. En esta ilustración se puede visualizar una alta frecuencia en el comportamiento de la volatilidad de la rentabilidad diaria de los criptoactivos, en el periodo 2019 a 2020 se verifican volatilidades moderadas.

Los resultados mostrados en la Tabla 3 hacen notar a Ethereum como el activo que tiene la más alta rentabilidad diaria, seguido de Bitcoin y más atrás están Ripple, Litecoin y Thether y la relación directa entre el riesgo y la rentabilidad, Ripple es el que tiene la más alta volatilidad en la rentabilidad diaria, seguido de Bitcoin y Ripple entre otros 4 activos que han intervenido en la conformación de la cartera de inversiones. Por otro lado, Ripple es el activo que ostenta la más alta volatilidad seguido de Ethereum, Litecoin y Bitcoin. Estos resultados obtenidos demuestran la hipótesis 1 de la investigación.

La Tabla 4 exhibe el resultado de la organización de 12 opciones de portafolio de inversiones. Se obtuvieron empleando el modelo de programación no lineal con función objetivo de maximización de la rentabilidad y minimización del riesgo de cada portafolio. Se han formulado las restricciones del modelo sobre el peso de cada componente del portafolio, buscando de entre las 12 opciones la eficiencia de la cartera, siendo aquella la cartera que exhiba el mayor índice de Sharpe.

Con la información mostrada en la Tabla 4 y obtenida mediante el modelo de programación no lineal que exhibe la rentabilidad, el riesgo y la composición de las 12 carteras

Tabla 3

Estadística descriptiva de la rentabilidad de criptomonedas

\begin{tabular}{lrrrrr}
\hline \multicolumn{1}{c}{ INDICADOR } & BITCOIN & ETHEREUM & LITECOIN & RIPPLE & THETER \\
\hline RENTABILIDAD MEDIA & 0.20 & 0.33 & 0.14 & 0.18 & 0.00 \\
VARIANZA & 15.30 & 37.33 & 29.92 & 42.27 & 0.48 \\
RIESGO & 3.91 & 6.14 & 5.47 & 6.50 & 0.69 \\
\hline
\end{tabular}

Fuente: Elaboración propia con base en Investing.com (2021)

Tabla 4

Organización de portafolios de inversión con criptomonedas

\begin{tabular}{ccccccccccc}
\hline PASOS & PORTAF & RIESGO & RENTABIL & BITCOIN & ETHREUM & LITECOIN & RIPPLE & THETHER & SUMA & SHARPE \\
\hline 0.5649 & MV & 0.6864 & 0.0004 & 0.0083 & 0.0020 & 0.0074 & 0.0061 & 0.9762 & $100 \%$ & 0.0003 \\
& 1 & 1.2513 & 0.0586 & 0.2234 & 0.0447 & 0.0000 & 0.0237 & 0.7083 & $100 \%$ & 0.0467 \\
& 2 & 1.8162 & 0.0936 & 0.3504 & 0.0705 & 0.0000 & 0.0334 & 0.5458 & $100 \%$ & 0.0515 \\
& 3 & 2.3811 & 0.1266 & 0.4701 & 0.0947 & 0.0000 & 0.0424 & 0.3927 & $100 \%$ & 0.0531 \\
& 4 & 2.9460 & 0.1589 & 0.5871 & 0.1185 & 0.0000 & 0.0514 & 0.2431 & $100 \%$ & 0.0539 \\
& 5 & 3.5109 & 0.1908 & 0.7027 & 0.1420 & 0.0000 & 0.0601 & 0.0952 & $100 \%$ & 0.0543 \\
& 6 & 4.0758 & 0.2191 & 0.7351 & 0.2649 & 0.0000 & 0.0000 & 0.0000 & $100 \%$ & 0.0537 \\
& 7 & 4.6407 & 0.2310 & 0.5195 & 0.4805 & 0.0000 & 0.0000 & 0.0000 & $100 \%$ & 0.0497 \\
& 8 & 5.2056 & 0.2394 & 0.3673 & 0.6327 & 0.0000 & 0.0000 & 0.0000 & $100 \%$ & 0.0460 \\
& 9 & 5.7705 & 0.2467 & 0.2356 & 0.7644 & 0.0000 & 0.0000 & 0.0000 & $100 \%$ & 0.0427 \\
& 10 & 6.3354 & 0.2534 & 0.1146 & 0.8854 & 0.0000 & 0.0000 & 0.0000 & $100 \%$ & 0.0400 \\
& MAX & 6.9003 & 0.2597 & 0.0000 & 1.0000 & 0.0000 & 0.0000 & 0.0000 & $100 \%$ & 0.0376 \\
& & & & & & & & & \\
& MAX IS & 3.5109 & 0.190756 & $70 \%$ & $14 \%$ & $0 \%$ & $6 \%$ & $10 \%$ & $100 \%$ & 0.0543 \\
\hline
\end{tabular}

Fuente: Elaboración propia con base en Investing.com (2021) 
de inversión organizadas con criptoactivos, se obtuvo la curva de la frontera eficiente que se exhibe en la Figura 3.

Dentro de las 12 opciones del portafolio, de acuerdo con la ecuación de Sharpe, la cartera óptima se ubica cuando se invierte $70 \%$ en Bitcoin, $14 \%$ en Ethereum, $6 \%$ en Ripple y $10 \%$ en Theter que corresponde al portafolio 5, que de acuerdo con el postulado de Sharpe es calificada como la cartera óptima; sin embargo, dependiendo del perfil de riesgo de los inversionistas, podrían asumir la composición de cartera que mejor calce con sus expectativas en términos de combinación de riesgo y rentabilidad. Algunos inversionistas podrían asumir mayores riesgos buscando obtener una mayor rentabilidad y, por otro lado, podrían haber inversionistas que optarían por una posición corta en riesgos buscando una menor rentabilidad que calce mejor con sus expectativas de logros esperados.

Los resultados mostrados en la Figura 4 exhiben el efecto de reducción del riesgo mediante la diversificación mediante

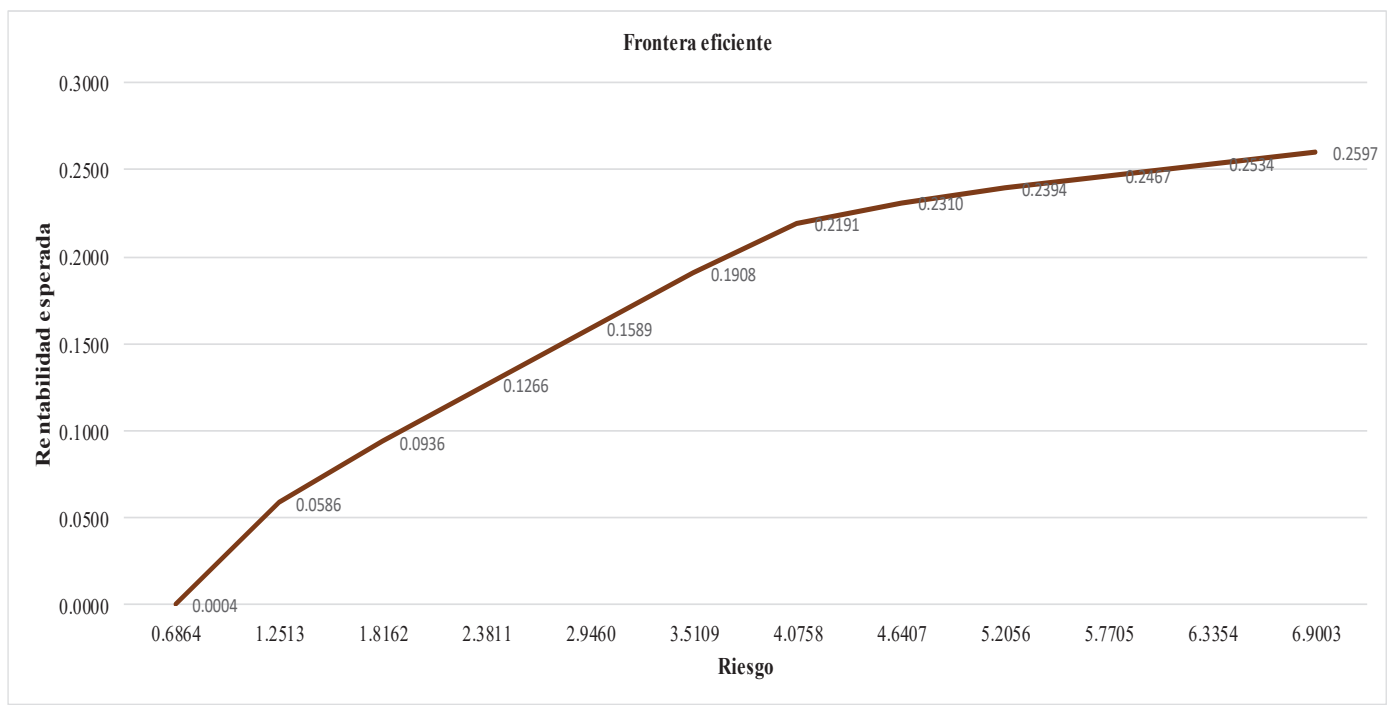

Figura 3. Frontera eficiente de portafolios de inversión con criptomonedas

Fuente: Elaboración propia con base en Investing.com (2021)

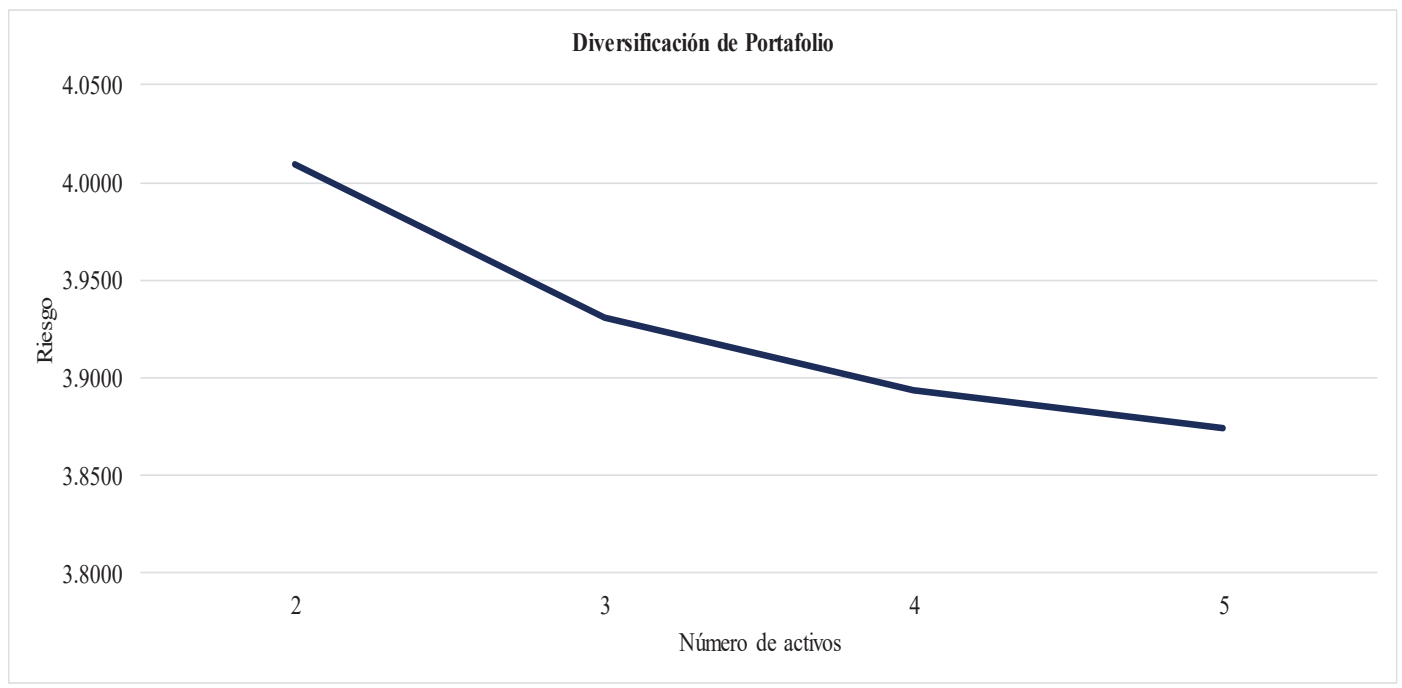

Figura 4. Diversificación de inversiones con criptoactivos

Fuente: Elaboración propia con base Investing.com (2021) 
la inclusión del número de activos en los portafolios de inversión. Así, se demuestra a través de un proceso experimental de agregar cada vez un mayor número de activos en el portafolio de inversiones, obteniendo como resultado el efecto de reducción de riesgo diversificable para los portafolios de inversión tal cual se puede verificar en la citada ilustración; así por ejemplo, en una cartera organizada con 2 activos se tiene el riesgo de 4\%; ante una cartera organizada con 3 activos, el riesgo es de $3.93 \%$ y frente a una cartera estructurada con 5 activos se tiene el riesgo de $3.87 \%$, con lo que queda demostrada la hipótesis de investigación número 2 .

\section{DISCUSIÓN}

De acuerdo con la teoría cuantitativa de organización de carteras de inversión de Markowitz (1959), es posible evaluar diversas alternativas de inversión con criptoactivos bajo la consideración típica del comportamiento de activos de renta variable, combinando, por un lado, el peso de los componentes de un portafolio de inversiones y con el número de activos que intervienen en una cartera de inversiones. En concordancia con esta teoría cuantitativa, los resultados obtenidos en la experiencia en cuestión, tal cual se visualizaron en la Figura 3, muestran la frontera eficiente que resulta de la combinación de pesos de cada uno de los componentes del portafolio de inversiones bajo el enfoque del modelo de Sharpe (2003), del cual se obtiene la mejor combinación de la cartera, que bien podría ser elegido por un potencial inversionista y, por otro lado, se propone otras combinaciones de riesgo y rendimiento.

Además, en la Figura 4, se mostró el efecto de la diversificación del portafolio de inversiones. Para esta experiencia, se organizaron 4 portafolios: el primer portafolio con 2 activos, el segundo con 3 activos, el tercero con 4 activos y el quinto con 5 activos. De cada experiencia se obtuvo la información del riesgo del portafolio, confirmando que, ante la presencia de un mayor número activos dentro de una cartera, se observa reducida la volatilidad de la cartera de inversiones, haciendo notar el impacto del número de componentes del portafolio en el nivel de volatilidad del portafolio de inversiones. En este mismo sentido, se han constatado los resultados de Sharma (2017) en la construcción y evaluación de carteras de inversión con criptomonedas bajo la teoría cuantitativa de Markowitz. Por otro lado, Liu et al. (2020) mostraron el impacto de la volatilidad en los criptoactivos y su incidencia en la rentabilidad de la cartera. En el presente estudio se confirma la relación implícita que existe entre la alta volatilidad del portafolio y una mayor rentabilidad buscada por parte de los inversionistas con sesgo en asumir altos riesgos en sus decisiones de inversión.
Los investigadores Can y Lagasse (2019), atraídos por el interés que habían despertado sobre la inclusión de criptomonedas en portafolios de inversión, han estudiado el papel de las criptomonedas en los portafolios de inversión, identificando la mejora de rendimiento del portafolio ante un mayor nivel de riesgo. Por otro lado, Jang y Lee (2018), por la importancia que venía despertando las inversiones en criptomonedas, estudiaron los métodos de pronóstico del Bitcoin mediante modelos de redes neuronales. En la dirección de las investigaciones citadas, el presente estudio ha constatado que existe una relación directa entre la rentabilidad y el riesgo, así como una relación indirecta entre el número de componentes del portafolio y su nivel de riesgo.

Lopes (1987) estudió la psicología del riesgo, al analizar la toma de decisiones de inversión bajo situaciones de riesgo. En el presente estudio, el escenario de la toma de decisiones por algunas de las opciones del portafolio expresado en la frontera eficiente ocurriría bajo situaciones de riesgo. Por otro lado, la frontera eficiente expuesta en la Figura 3 de entre los 12 portafolios muestra una que satisfice el postulado de cartera eficiente de Sharpe (2003).

Los resultados encontrados en el presente estudio son de singular importancia para la organización de portafolios de inversión con activos financieros porque confirman el impacto de la diversificación en la reducción del riesgo del portafolio. En este caso específico, el impacto del número de activos en la minimización del riesgo de un portafolio de inversiones y una mezcla de pesos y activos que conducen a encontrar mejores alternativas de inversión mostradas en la frontera eficiente, organizadas mediante el algoritmo de optimización que proveen a los potenciales inversionistas diversas opciones sobre la elección de mejores alternativas que calcen con sus expectativas de rentabilidad esperada y riesgo aceptado.

Como conclusiones que derivan del estudio realizado, se puede remarcar la necesidad de tener presente el concepto de diversificación en la construcción de portafolios de inversión con el fin de maximizar la rentabilidad y minimizar el riesgo. Por otro lado, se denota de manera muy especial que el número de activos que intervienen en la conformación de activos en los portafolios contribuyen en la reducción de la volatilidad de la cartera.

En relación con la organización de carteras de inversión con activos digitales, la agenda futura de investigación referida al tema debería responder a las siguientes cuestiones: ¿Cómo incluir los activos digitales en la conformación de carteras con activos de renta variable y commodities?, y ¿es necesario estudiar sobre la construcción de mercados organizados en donde estén incluidos los criptoactivos? 


\section{REFERENCIAS}

Andrianto, Y., \& Diputra, Y. (2018). The Effect of Cryptocurrency on Investment Portfolio Effectiveness. Journal of Finance and Accounting, 5(6), 229-238.

Can, A., \& Lagasse, R. (2019). Cryptocurrencies: applications and investment opportunities. Journal of Capital Markets Studies, 3(2), 98-112.

Copeland, T., \& Weston, J. (1988). Financial Theory and Corporate Policy. New York: Addison Wesley Publishing Company.

Cortez, A., \& Tulcanaza, A. (2018). Bitcoin: Its Influence on then Global world and Its Relationship with the Stock Exchange. Journal Of Chakiñan, 54-72.

Damodaran, A. (2012). Investment Evaluation: Tools and Techniques for Determining the Value of Any Asset. New Jersey: Wiley Finance.

Holovatiuk, O. (2020). Cryptocurrencies as an asset class in portfolio optimization. Central European Economic Journal, 7(54), 33-55.

Investing.com. (2021). Repositorio de base de datos de criptomonedas. Recuperado de: https://www.investing.com/
Jang, H., \& Lee, J. (2018). An Empirical Study on Modeling and Prediction of Bitcoin Prices with Bayesian Neural Networks Based on Blockchain Information. IEEE Access. Recuperado de: http:/www.ieee.org/publications_stndards/publications/rights/index.html

Liu, M., Wang, Z., Ahmad, F., \& Ma, Y. (2020). Portfolio optimization in the era of digital financialization using cryptocurrencies. Journal of Technological Forecasting \& Social Change. doi.org/J.techfore.2020.120265.

Lopes, L. (1987). Between Hope and Fear: The Psychology of Risk. Advances in Experimental Social Psychology, 20, 255-295.

Markowitz, H. (1959). Portfolio Selection: Efficient Diversification of Investments. Massachusetts: Wiley.

Scimago Journal \& Country Rank (2020). Journal rankings. Recuperado de: https://www.scimagojr.com/ journalrank.php?area $=2000$

Sharpe, A., \& Bailey, J. (2003). Investment Fundamentals: Theory and Practice. New York: Prentice Hall Inc.

Sharma, A. (2017). Build a Portfolio of Cryptocurrencies using Modern Portfolio Theory. Recuperado de: https://medium.com/build-of-cryptocurrencies-using-modern-portfolio-theory 